\title{
GA-tuning of Nonlinear Observers for Sensorless Control of Automotive Power Steering IPMSMs
}

\author{
B. S. Bhangu (MIEEE), C. M. Bingham (MIEEE) \\ Department of Electronic and Electrical Engineering \\ University of Sheffield, Mappin Street, \\ Sheffield, S1 3JD, UK. \\ Corresponding author: b.bhangu@sheffield.ac.uk
}

\begin{abstract}
The paper considers two observer-based rotor position estimation schemes for sensorless control of Interior Permanent Magnet Synchronous Motors (IPMSMs) for use in future automotive power steering systems. Specifically, emphasis is given to techniques based on feedback-linearisation followed by classical Luenberger observer design, and direct design of non-linear observers. Genetic Algorithms (GAs), using the principles of evolution, natural selection and genetic mutation, are introduced to address difficulties in selecting correction gains for the observers, since no analytical tuning mechanisms yet exist. Experimental measurements from an automotive power steering test-facility are included, to demonstrate the enhanced performance attributes offered by tuning the proposed observer schemes, online, in this manner.
\end{abstract}

Index Terms- Genetic algorithms, Nonlinear estimation, Observers, Permanent magnet machines, State estimation.

\section{INTRODUCTION}

$\mathrm{I}_{\mathrm{in}}^{\mathrm{n}}$ ncreasing pressure from governments, and other bodies, to improve vehicle fuel economy and reduce carbon emissions, has lead to automotive manufacturers targeting auxiliary vehicle power systems as a means of improving efficiency. The use of power assisted steering systems, in particular, is now a relatively standard feature of most massproduced domestic vehicles, and improved efficiency could yield marked reductions in the vehicle auxiliary load, and consequently, promote reduced vehicle emissions. By way of example, for a typical hydraulic power steering system the resulting load on the vehicle is around $1.5 \mathrm{~kW}$, of which only $0.5 \mathrm{~kW}$ may be transformed into mechanical assistance at the steering shaft. The resulting system efficiency is therefore relatively low. It is estimated that the result of employing electric-based power assistance is equivalent to an improvement in fuel economy of up to $4 \%$ [1].

\section{ELECTRIC POWER ASSISTED STEERING}

Two generic types of electric assisted steering systems are commonly employed by the automotive sector viz. full Electric Power Steering (EPS), and electrically powered hydraulic steering (EPHS); a combination of electric and hydraulic units, as schematically depicted, respectively, in Figs. 1(a) and (b). Such systems generally have three modes of operation:

i. to assist the driver to make a controlled turn

ii. to assist the driver to return to a straight line after making a turn

iii. improve road feel and to dampen kickback.

Additionally, on the occurrence of a full-lock steering demand, the Electronic Control Unit (ECU) reduces the supply current to the motor to prevent overload conditions, and, upon the occurrence of a fault, must also ensure that the system fails to a safe state i.e. reverts to manual steering.

EPS systems employ electromechanical actuation, with an electric motor attached to the steering rack or column via a gear mechanism, as opposed to the more traditional hydraulic pump. Sensors located on the input shaft determine the amount of torque the driver is applying on the steering wheel, the direction and speed of the steering wheel, and steering angle. These measurements, along with the vehicle velocity are fed into the on-board ECU microprocessor, where lookup tables determine the required amount of power assistance. The resulting demand is fed to the drive circuitry to appropriately excite the bi-directional motor, pushing the rack in either direction.

Primary advantages of EPSs are the elimination of traditional hydraulic elements, such as the pumps, fluids, hoses, pulleys and drive-belts. Furthermore, their operation is engine independent, thereby reducing engine drain (giving improved vehicle acceleration) and allowing assistance even when the engine is off - the latter issue being particularly useful for hybrid power trains where the engine is often turned-off and tractive power is delivered by pure electric means. 


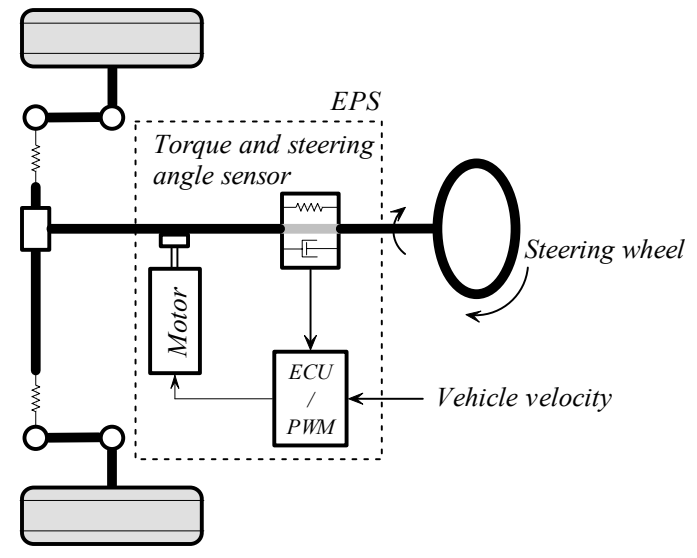

(a)

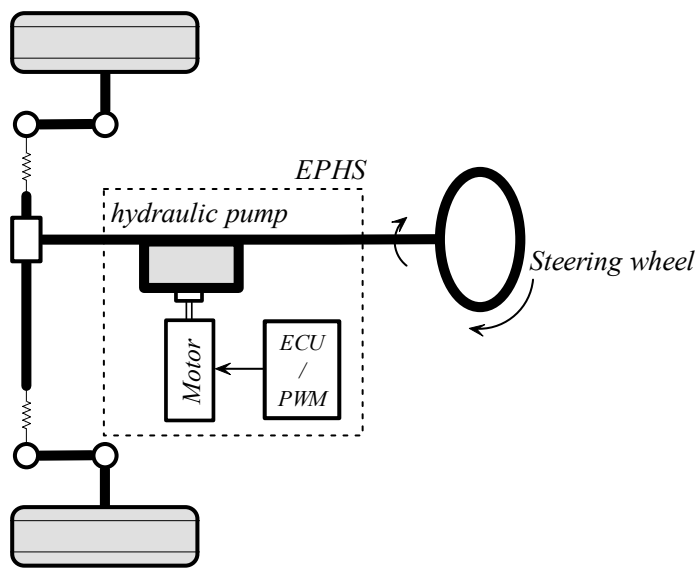

(b)

Fig. 1. Schematic of electric-based power assisted steering systems (a) EPS system (b) EPHS system.

A variant lying between conventional hydraulic steering technologies, and full electric integration, is EPHS. As with EPS systems, all direct connections to the engine are eliminated in favour of using an electric motor, ECU and hydraulic pump, all constructed as an integrated unit that is readily linked to a conventional rack and pinion gear to provide steering assistance when needed. Assistance is obtained by regulating the pump via control of the electric motor to provide a desired pressure and flow-rate. To provide energy savings, the pump is able to operate at low speed, or shutdown, when the car is travelling in a straight line. The main advantages offered by current EPHS systems are enhanced performance at reduced cost.

\section{PM MOTOR / DRIVE REQUIREMENTS}

Primary in-service handling requirements of all power assisted steering systems are to impart smooth torque transfer to the mechanical steering linkages whilst also providing a degree of torque feedback to the driver.

Existing EPHSs normally consist of a Permanent Magnet Brushless-DC (PMBDC) machine that continuously circulates hydraulic fluid for power steering assistance, whilst running at a low standby speed. However, it is notable that PMBDC machines, by virtue of their 2-phase-on block commutation, can cause relatively high acoustic noise emissions and torque ripple. With the foreseeable adoption of increasing numbers of brushless machines in the vehicle, audible noise is becoming a concern, and PM Brushless-AC motor (PMBAC) counterparts are therefore becoming preferred candidates. For sinusoidal commutation, however, encoders (and Hall-effects to facilitate full-torque start-up) or resolvers are traditionally required. Such sensors decrease reliability (due to being subjected to harsh environments) and usually require custom mounting arrangements. In addition, intense economic constraints, product volumes and manufacture/motor parameter tolerance issues, consistent with the requirements of vehicle technology industries, have meant that the cost and component count attributed to the requirement for dedicated rotor position sensors for the increasing number of PMSMs envisaged for auxiliary systems, is leading to greater interest in sensorless rotor position estimation schemes. Furthermore, where applications call for custom motor designs, the omission of a rotor position sensor has the added benefit of increasing the active length of the rotor/stator, thereby further increasing the power/torque obtained for a given volume envelope.

Nevertheless, before any candidate sensorless solution can be considered appropriate, the automotive sector requires that the following key issues be addressed:

i. Minimal algorithm complexity/code length

ii. Accurate rotor position estimates during dynamic load disturbances - no transient loss of information

iii. Robustness to parameter tolerances

iv. Low acoustic noise emissions.

The first issue relates to the economics of utilising extra capacity on existing minimal-cost computational hardware (there is little point in replacing position sensors with relatively expensive processing hardware), whilst the next two points concern operational performance of the motor, and the consequences of volume manufacture and changes in motor parameters due to the ambient thermal operating envelope (typically -40 to $125^{\circ} \mathrm{C}$ ), respectively. Finally, the $4^{\text {th }}$ issue reflects requirements for user comfort in the cabin.

\section{OBSERVER-BASED SENSORLESS CONTROL}

For servo-type applications, and those where electromagnetic torque ripple and audible noise have a significant impact on overall product quality, vector control schemes are preferred [2]. In such cases, sensorless operation can only be achieved by utilising motor terminal quantities to estimate rotor position.

The situation is further complicated for applications in the vehicular technology industry, where the use of Interior Permanent Magnet Synchronous Machines (IPMSMs), or buried magnet motors, where the magnets are mounted inside the rotor, are preferred Such machines have advantages for cost and performance-critical applications viz. the flat, buried magnets can be cheaper to manufacture and can produce 


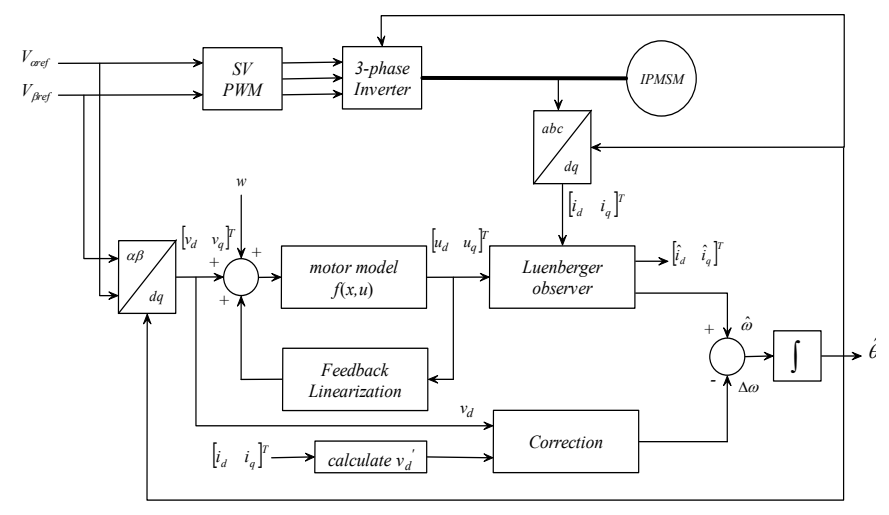

(a)

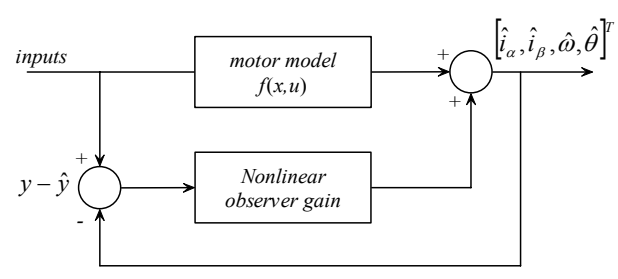

(b)

Fig. 2. Structure of observer schemes. (a) Feedback linearization and Luenberger observer (b) Nonlinear observer.

similar flux densities to surface mounted counterparts whilst requiring less bulk material, and, for high speed operation, the rotor possesses enhanced mechanical robustness since the rotor iron encapsulates the magnets. A consequence of using buried magnets, however, is that the direct and quadrature motor inductances are no longer equal, with $L_{d}$ and $L_{q}$ differing typically by between 5 and $30 \%$.

Although various observer-based sensorless techniques have been proposed for non-salient PMSMs [3,4,5,6,7,8,9], their application to salient machines, for addressing the specific requirements of automotive systems, remains outstanding.

Here then, two observer-based state-estimation techniques, are developed, specifically for application to IPMSMs, with the use of GAs to assist in selection of optimal observer gains. The structural difference between the proposed observer techniques, is shown in Fig. 2. The first method involves feedback linearization followed by the design and subsequent implementation of a classical Luenberger observer, extended to include salient nonlinearities, whilst the second proposes the direct design of a non-linear observer.

\section{IMPACT OF SALIENCY ON POSITION ESTIMATES}

Employing observers on buried magnet machines without accommodating the effects of saliency is known to significantly degrade the accuracy of resulting rotor position estimates, as demonstrated in [10]. Consequently, formulations specifically for application to such machines, must be made.

\section{A. Feedback Linearization and Luenberger Observer Design with Effects of Saliency}

A dynamic model of an IPMSM, in rotor-fixed d-q reference frame, is given in (1), where the applied inputs are the transformed terminal voltages $u=\left[v_{d}, v_{q}\right]^{T}$, and the measured outputs are the machine terminal currents $y=\left[i_{d}, i_{q}\right]^{T}$.

$\frac{d}{d t} i_{d}=-\frac{R_{s}}{L_{d}} i_{d}+\omega i_{q} \frac{L_{q}}{L_{d}}+\frac{v_{d}}{L_{d}}$

$\frac{d}{d t} i_{q}=-\frac{R_{s}}{L_{q}} i_{q}-\omega i_{d} \frac{L_{d}}{L_{q}}-\frac{K_{e}}{L_{q}} \omega+\frac{v_{q}}{L_{q}}$

$\frac{d}{d t} \omega=\frac{K_{t} p}{J} i_{q}+\frac{3 p}{2 J}\left(L_{d}-L_{q}\right) i_{d} i_{q}-\frac{B}{J} \omega$

$\frac{d}{d t} \theta=\omega$

From (1), it is evident that equations describing the electrical dynamics are coupled by nonlinear angular velocity terms. The classical use of feedback linearization methods [5] means that such nonlinearities can be accommodated through the use of auxiliary inputs:

$u_{d}=v_{d}+\omega i_{q} L_{q}$

$u_{q}=v_{q}-\omega i_{d} L_{d}$

that employ nonlinear feedback of motor currents and angular velocity (estimated in practice). For a salient machine, the resulting dynamic description is given by:

$\frac{d}{d t} i_{d}=-\frac{R_{s}}{L_{d}} i_{d}+\frac{u_{d}}{L_{d}}$

$\frac{d}{d t} i_{q}=-\frac{R_{s}}{L_{q}} i_{q}-\frac{K_{e}}{L_{q}} \omega+\frac{u_{q}}{L_{q}}$

$\frac{d}{d t} \omega=\frac{K_{t} p}{J} i_{q}+\frac{3 p}{2 J}\left(L_{d}-L_{q}\right) i_{d} i_{q}-\frac{B}{J} \omega$

$\frac{d}{d t} \theta=\omega$

However, from (3), it is evident that a non-linear term remains by virtue of the non-zero term $\left(L_{d}-L_{q}\right)$ that is present as a consequence of motor saliency, thereby preventing classical Luenberger-type observer techniques to be subsequently applied - as is commonplace with the nonsalient motor counterparts [10].

A proposed solution is, therefore, to introduce an additional non-linear state, $z$, such that:

$z=i_{d} i_{q}$

and, from (3), the time-derivative is given by:

$\frac{d}{d t} z=-R_{S}\left(\frac{1}{L_{d}}+\frac{1}{L_{q}}\right) z+\frac{U_{z 1}}{L_{d}}+\frac{U_{z 2}}{L_{q}}$

where,

$U_{z 1}=u_{d} i_{q}$

$U_{z 2}=-K_{e} \omega i_{d}+u_{q} i_{d}$

resulting in the new dynamic description: 


$$
\frac{d}{d t}\left[\begin{array}{c}
i_{d} \\
i_{q} \\
\omega \\
z
\end{array}\right]=\left[\begin{array}{c}
-\frac{R_{s}}{L_{d}} i_{d}+\frac{u_{d}}{L_{d}} \\
-\frac{R_{s}}{L_{q}} i_{q}-\frac{K_{e}}{L_{q}} \omega+\frac{u_{q}}{L_{q}} \\
\frac{K_{t} p}{J} i_{q}+\frac{3 p}{2 J}\left(L_{d}-L_{q}\right) z-\frac{B}{J} \omega \\
-R_{s}\left(\frac{1}{L_{d}}+\frac{1}{L_{q}}\right) z+\frac{U_{z 1}}{L_{d}}+\frac{U_{z 2}}{L_{q}}
\end{array}\right]
$$

Since $U_{z 1}$ and $U_{z 2}$ are dependent on the auxiliary inputs and the terminal currents, which will be measured quantities, they can be considered as known disturbances. Hence, a state variable representation of the machine, suitable for the design of a Luenberger observer, can be derived from (7), as follows,

$$
\begin{aligned}
\frac{d}{d t}\left[\begin{array}{c}
i_{d} \\
i_{q} \\
\omega \\
z
\end{array}\right] & {\left[\begin{array}{cccc}
-\frac{R_{S}}{L_{d}} & 0 & 0 & 0 \\
0 & -\frac{R_{s}}{L_{q}} & -\frac{K_{e}}{L_{q}} & 0 \\
0 & \frac{K_{t} p}{J} & -\frac{B}{J} & -\frac{3 p}{2 J}\left(L_{d}-L_{q}\right) \\
0 & 0 & 0 & -R_{s}\left(\frac{1}{L_{d}}+\frac{1}{L_{q}}\right)
\end{array}\right]\left[\begin{array}{l}
i_{d} \\
i_{q} \\
\omega \\
z
\end{array}\right] } \\
& +\left[\begin{array}{cc}
\frac{1}{L_{d}} & 0 \\
0 & \frac{1}{L_{q}} \\
0 & 0 \\
0 & 0
\end{array}\right]\left[\begin{array}{l}
u_{d} \\
u_{q}
\end{array}\right]+\left[\begin{array}{cc}
0 & 0 \\
0 & 0 \\
0 & 0 \\
\frac{1}{L_{d}} & \frac{1}{L_{q}}
\end{array}\right]\left[\begin{array}{l}
U_{z 1} \\
U_{z 2}
\end{array}\right]
\end{aligned}
$$$$
\text { or }
$$

$$
\dot{x}=\mathbf{A} x+\mathbf{B} u+w
$$

where $x=\left[\begin{array}{llll}i_{d} & i_{q} & \omega & z\end{array}\right]^{T}$

The structure of the resulting observer is given by:

$$
\dot{\hat{x}}=\mathbf{A} \hat{x}+\mathbf{B} u+w+\mathbf{K}(y-\hat{y})
$$

where $w$ is the 'known' disturbance given by last term on RHS of (8).

It is notable that the state variable relating to $\theta$ has been omitted in (7-9), in favour of utilising an auxiliary correction feature, initially proposed in [11], to account for the unobservability of $\theta$, as a result of the feedback linearization process given by (2). The complete rotor position estimation scheme therefore consists of

$$
\begin{aligned}
& \text { i. a feedback linearising controller } \\
& \text { ii. a linear observer } \\
& \text { iii. } \quad \text { a correction scheme to allow } \hat{\theta} \text { to converge } \\
& \text { to } \theta
\end{aligned}
$$

as shown in Fig. 2(a). Ultimately, the performance of the observer is dependent on the assignment of suitable convergence dynamics, via the choice of $\mathbf{K}$, and is motor parameter dependent. The eigenvalues of the observer are generally assigned to be of sufficiently high-bandwidth, compared to the motor dynamics, so as to minimise the effects of a transient convergence of state estimates on closed-loop speed and position control systems. Specifically, the error convergence dynamics are designed by noting the difference between the system state vector, and the observed state vector $(x-\hat{x})$ and are governed by [12]:

$\dot{e}=\dot{x}-\dot{\hat{x}}=(\mathbf{A}-\mathbf{K C}) e$

where $\mathbf{K}$ assigns appropriate eigenvalues.

The performance of the proposed observer is demonstrated on a closed-loop, speed controlled, power-steering testfacility, Fig. 3, incorporating a, $0.7 \mathrm{~kW}, 12 \mathrm{~V}$ IPMSM with $28 \%$ saliency. In this case, the electrical time constant of the motor is $3.5 \mathrm{~ms}$ and the inertia of the motor and load is $1.7 \times 10^{-4} \mathrm{kgm}^{2}$.

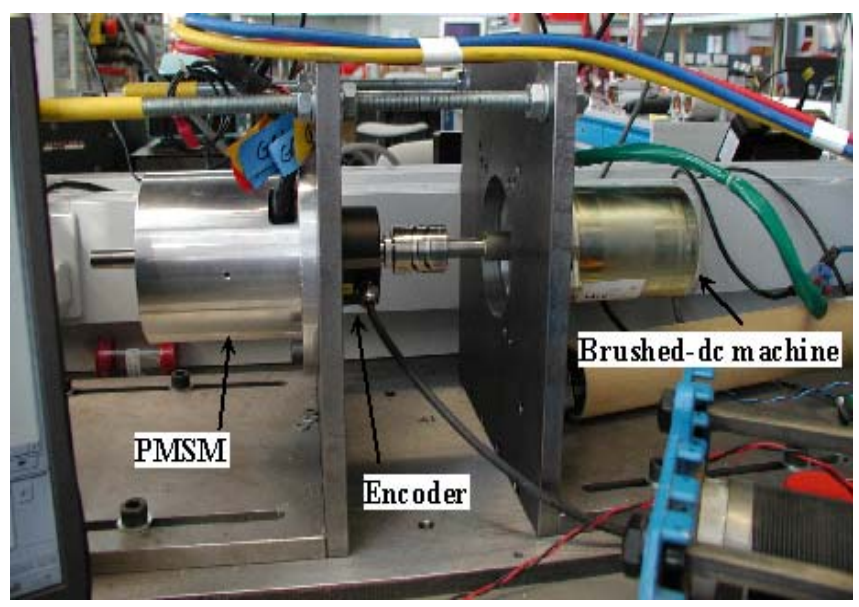

Fig. 3. Power assisted steering test facility.

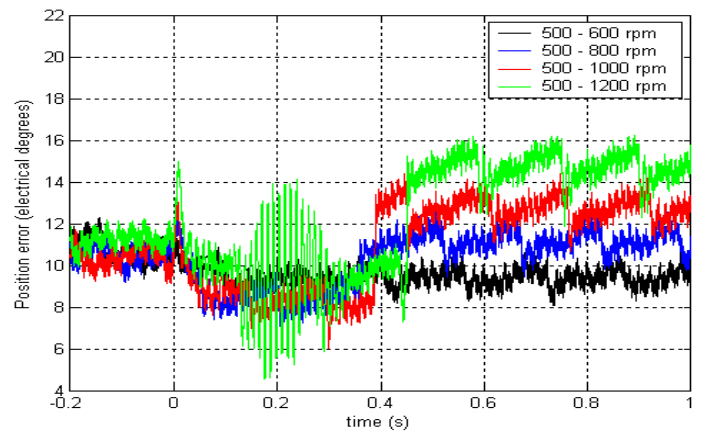

(a)

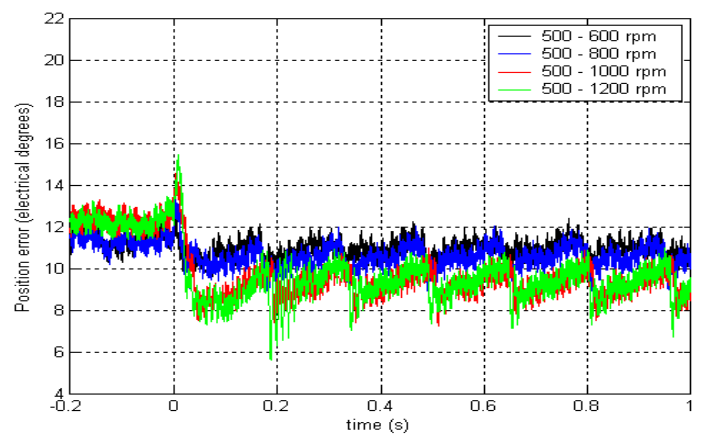

(b)

Fig. 4. Power assisted steering test facility. Rotor position error in response to transient speed demands at $\mathrm{t}=0 \mathrm{~s}$. (a) Non-salient Luenberger observer (b) Salient Luenberger observer. 
Figure 4 shows the performance resulting from application of the non-salient observer scheme, and the proposed salient variant, respectively, in response to various transient step demands from $500 \mathrm{rpm}$ at $\mathrm{t}=0 \mathrm{~s}$. Neglecting the effects of saliency in the observer, Fig. 4(a) shows the increase in steady-state position error. However, by integrating the effects of the salient dynamics into the observer, as discussed, along with the known disturbance, $w$, improvements in the rotor position estimates are evident from the measurements given in Fig. 4(b), particularly for relatively high speed demands. Although position errors are present in all cases, a general improvement in both the dynamic- and steady-state responses can be seen when the salient observer variant is employed. Moreover, when using the non-salient observer, a general reduction in the robustness of estimate convergence, is also evident, with unstable tendencies being present for large transient demands.

\section{B. Non-linear observer scheme incorporating the effects of saliency}

A full account of the formulation of the proposed direct nonlinear observer, is beyond the scope of this paper. The reader is therefore re-directed to [4] for derivation details and to [12] for full details of the salient counterpart. Here then, for brevity, the resulting structure of the proposed salient observer is merely stated as:

$$
\begin{aligned}
\frac{d}{d t}\left[\begin{array}{c}
\hat{i}_{\alpha} \\
\hat{i}_{\beta} \\
\hat{\omega} \\
\hat{\theta}
\end{array}\right]= & {\left[\begin{array}{c}
-\frac{R_{s}}{L_{d}} i_{\alpha}+\frac{K_{e}}{L_{d}} \omega \sin \theta-\omega L_{o} i_{q} \cos \theta-i_{q} L_{o} \sin \theta+\frac{v_{\alpha}}{L_{d}} \\
-\frac{R_{s}}{L_{d}} i_{\beta}-\frac{K_{e}}{L_{d}} \omega \cos \theta-\omega L_{o} i_{q} \sin \theta+\dot{i}_{q} L_{o} \cos \theta+\frac{v_{\beta}}{L_{d}} \\
\frac{K_{t} p}{J}\left(\hat{i_{\beta}} \cos \hat{\theta}-\hat{i}_{\alpha} \sin \hat{\theta}\right)+\frac{3 p}{2 J}\left(L_{d}-L_{q}\right) i_{d} i_{q}-\frac{B}{J} \hat{\omega} \\
\hat{\omega}
\end{array}\right] } \\
+\mathbf{K}(y-\hat{y}) &
\end{aligned}
$$

where, the observer gain $\mathbf{K}$ takes the form,

$$
\begin{aligned}
& \mathbf{K}=\left[\begin{array}{c}
K_{y} \\
\Gamma_{s} K_{z}
\end{array}\right], \quad K_{y}=\left[\begin{array}{cc}
K_{y 1} & 0 \\
0 & K_{y 2}
\end{array}\right] \\
& \Gamma_{s}=\frac{L_{d}}{K_{e} \omega}\left[\begin{array}{cc}
\omega \sin \theta & -\omega \cos \theta \\
\cos \theta & \sin \theta
\end{array}\right], \quad K_{z}=\left[\begin{array}{cc}
K_{z 1} & 0 \\
0 & K_{z 2}
\end{array}\right] \\
& L_{0}=\frac{L_{d}-L_{q}}{L_{d}}
\end{aligned}
$$

Notably, no formal tuning methods have yet been reported for selecting $K_{y}$ and $K_{z}$, and recourse to either 'sweepparameter' methods or intelligent tuning methods is ultimately required.

Example measurements comparing the performance of both the salient and non-salient observer schemes, is given in Fig. 5 , where step changes in speed demand, from 500 to 1000 rpm, are applied. It can be seen that the proposed salient observer scheme consistently outperforms the non-salient counterpart, with $40 \%$ improvement in position error being evident. The gain matrices in each case depicted here have been 'tuned' on-line by empirical means.

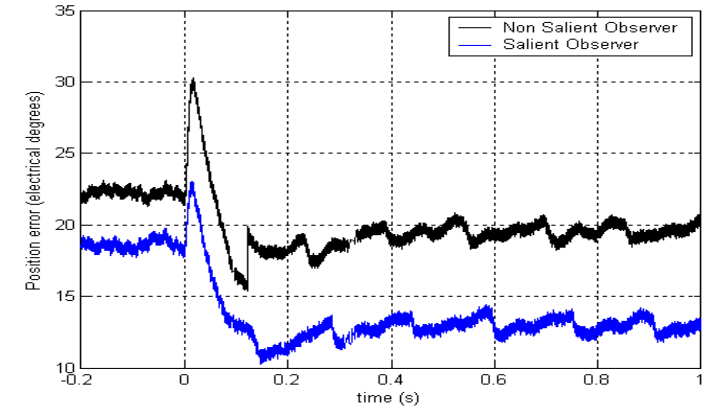

Fig. 5. Position error in response to a step speed demand at $\mathrm{t}=0 \mathrm{~s}$.

\section{GA TUNing OF THE NONLINEAR OBSERVE}

Two methods for choosing the elements of the gain matrices, are now investigated. The first, based on a 'parameter sweep', merely scans elemental values of the gain matrices and performs tests to determine the resulting position error. The second technique is an 'intelligent' tuning method based on the on-line implementation of a Genetic Algorithm (GA).

\section{A. Parameter Sweep Method}

Simulation studies have been conducted to identify regions of the gain parameter space that facilitate stable sensorless control of the IPMSM. Fig. 6(a) shows an example result depicting the stable and unstable regions of the resulting closed-loop system, for a range of gain values - the stable and unstable regions are classified by plotted values ' 0 ' and ' 1 ', respectively. All results are obtained at steady-state operating conditions at 1000rpm.

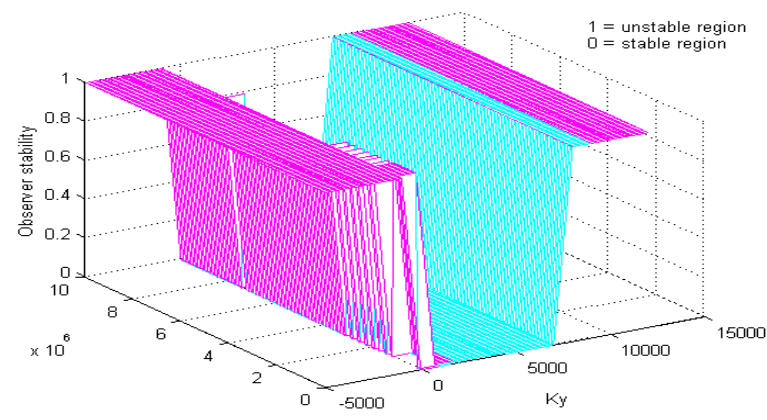

(a)

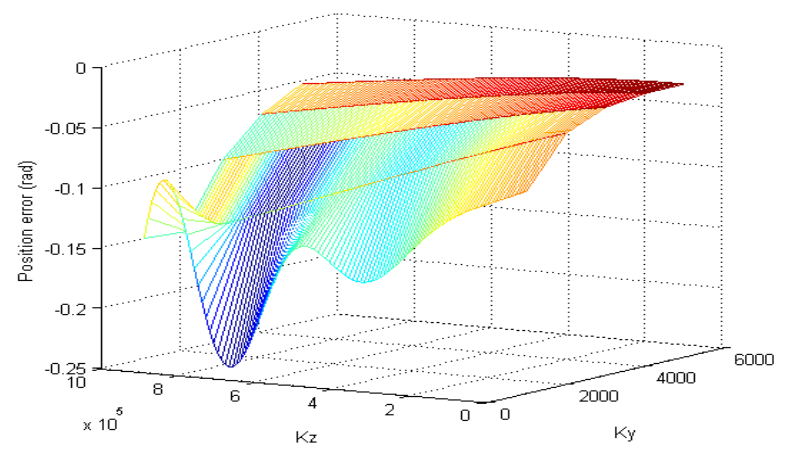

(b)

Fig. 6. Simulation results from a parameter sweep of correction gains. (a) Stable and unstable region (b) Stability and performance for a range of $K_{y}$ and $K_{z}$. 
On closer examination of the results from the stable region, Fig. 6(b) shows the resulting steady-state position error for the associated range of $K_{y}$ and $K_{z}$. In this case, it is seen that performance is enhanced when $K_{y}$ is bounded between $4000 \rightarrow 6000$, and $K_{z}$ between $0.5 \rightarrow 1 \times 10^{6}$, and that, by appropriate choice, the position error can be within $0.03 \mathrm{rad}$ (elec).

\section{B. Genetic Algorithm Implementation}

An alternative technique is to select observer gains based on the results from a GA, tuned on-line. A block diagram representation of the basic operation of the GA, with the objective of obtaining minimum position error, is shown in Fig. 7. In principle, 'parents' are selected for reproduction with a bias towards individuals with a 'high-fitness', and reproduced by cross breeding or recombining by processes of crossover and mutation [13] to generate off-spring. Repetition of this process leads to evolutionary populations that enhance the fitness function [13] (position error in this case).

Results from the candidate IPMSM, Fig. 8(a), shows an example GA trial exploring a large state-space to maximise the fitness function i.e. minimum rotor position error, with Fig. 8(b) showing the rotor position error obtained from initial observer gains (obtained from the 'sweep test' and trial-anderror basis) and with resultant gains obtained from on-line GA trials for a range of $K_{y}$ and $K_{z}$ from $0 \rightarrow 2000$. It is evident that the GA selects gains that generally provide smaller transient overshoot, lower settling time and reduced steady state error.

The following accounts the experimental implementation of the GA, on-line, to tune the nonlinear observer gains for enhanced sensorless performance of the vector controlled IPMSM. A performance comparison of position error at various speeds, is given in Fig. 9. The parameter sweep method, along with the best 'manual' tuning of observer, and the associated steady-state position error at various steadystate operating speeds, is shown in Fig. 9 (a). It can be seen that the observer gains obtained from the use of the GAs result in similar characteristics, but with reduced position error when compared to the results of the sweep tests. Due to the statistical nature of GA evolution, however, it is likely that different sets of gains will result from each trial (in practical situations at least, since the global optima is likely to be slightly different due to changes in operating temperature, for instance, and other extraneous effects). Figure 9 therefore also shows that although the observer gains from two independent tests, termed GA1 and GA2, each produced a different set of gains $(\approx 60 \%$ variation $)$, Table $\mathrm{I}$, whilst being tuned at a steady-state speed demand of $1000 \mathrm{rpm}$, it can be seen that the performance in each case is qualitatively very similar.

More generally, since it is recognised that the evolution of GAs are based on statistical features, extensive testing is often necessary to ensure the results do not become trapped within local minima, and provide a degree of confidence that solutions are optimal. Results of several trials, at various operating speeds, and with different statistical properties, in particular, by defining the probability of crossover and mutation (termed 'xov' and 'mut' respectively), are applied to the GA. From Fig. 10, which shows the resulting steady-state error after each set of gains has been applied to the observer, it can be seen that the performance is, again, qualitatively very similar each case, even with typical variations of $K_{y}$ and $K_{z}$ being $\approx 35 \%$ and $\approx 65 \%$, respectively. This is therefore suggestive that a characteristic of this system is that localised optima provide similar performance features.

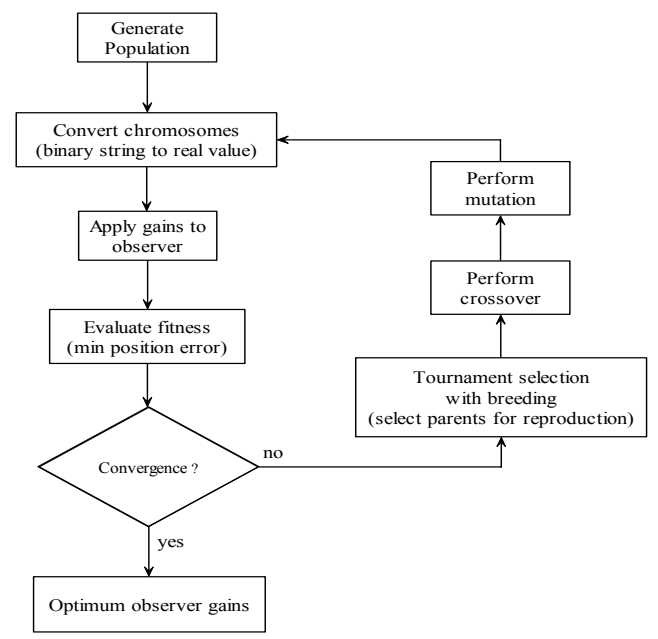

Fig. 7. GA for tuning nonlinear observer.

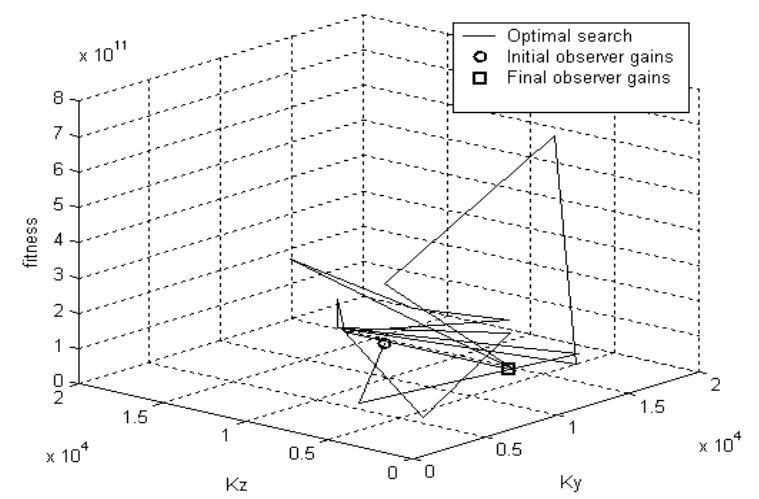

(a)

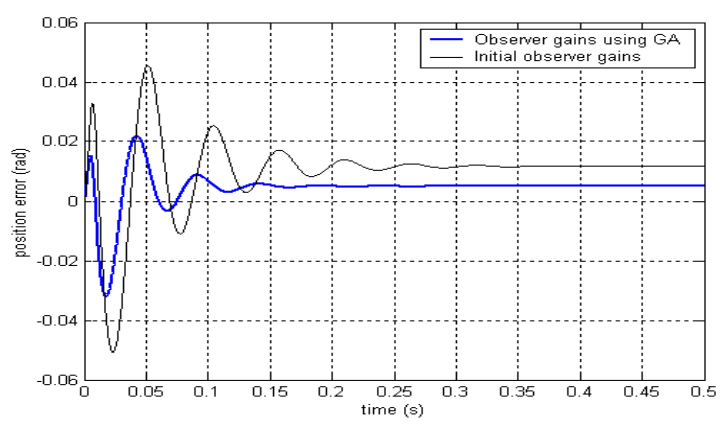

(b)

Fig. 8. Simulated study of parameter sweep gains to online implementation of GAs. (a) On-line selection of observer gains (b) Position error associated with using GA observer gains. 


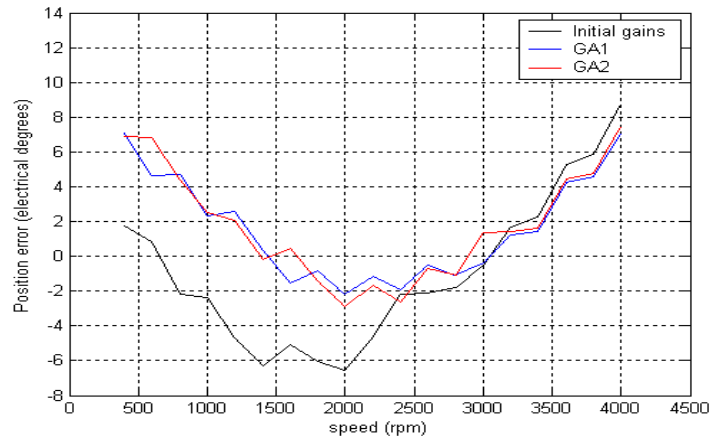

Fig. 9. Experimental results illustrating comparison of sweep test gains (Initial gains) to observer gains obtained from on-line GAs for GA1 and GA2 shown by Table I.

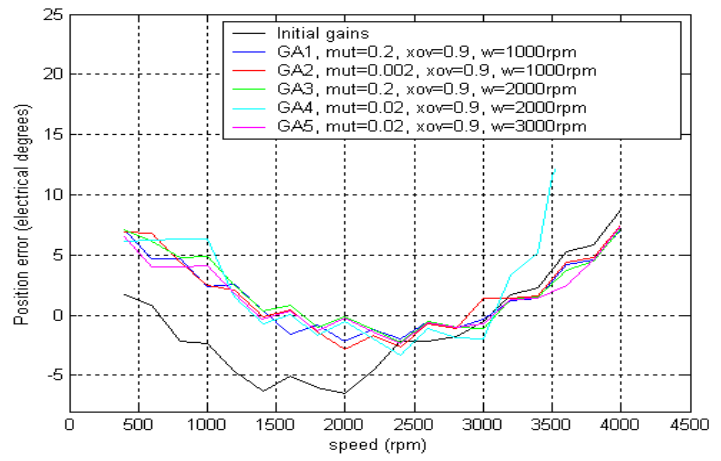

Fig. 10. Position error measurements from automotive salient machine for various operating speeds and statistical properties using optimal observer gains identified by GAs.

TABLE I

OBSERVER GAINS FROM SWEEP TEST AND GAs AT 1000RPM

\begin{tabular}{cccc}
\hline \hline Test & $K_{y 1}$ & $K_{z 1}$ & $K_{z 2}$ \\
Initial & 5000 & $1 \times 10^{6}$ & $1 \times 10^{6}$ \\
GA1 & 5000 & $7.8692 \times 10^{6}$ & $87.0799 \times 10^{6}$ \\
GA2 & 5000 & $17.8133 \times 10^{6}$ & $76.1199 \times 10^{6}$ \\
\hline \hline
\end{tabular}

TABLE II

PARAMETER VARIATION OF $R_{s}$ AND $K_{e}$.

\begin{tabular}{|c|c|c|c|c|}
\hline & & & $\boldsymbol{K}_{e}$ & \\
\hline \multirow{4}{*}{$\boldsymbol{R}_{s}$} & $\begin{array}{l}\text { Multiplying } \\
\text { factor }\end{array}$ & 0.89 & 1 & 1.1 \\
\hline & 0.8 & $K_{e}$ lo $\mathrm{R}_{s}$ lo & $\mathrm{R}_{s}$ lo & $K_{e}$ hi $\mathrm{R}_{s}$ lo \\
\hline & 1 & $K_{e}$ lo & 1 & $K_{e}$ hi \\
\hline & 1.21 & $K_{e}$ lo $\mathrm{R}_{s}$ hi & $\mathrm{R}_{s}$ hi & $K_{e}$ hi $\mathrm{R}_{s}$ hi \\
\hline
\end{tabular}

TABLE III

OBSERVER RESOURCE REQUIREMENTS

\begin{tabular}{ccc}
\hline \hline & Computation time & Memory requirement \\
EKF & 1 & 1 \\
Luenberger & 0.10 & 0.34 \\
Nonlinear & 0.17 & 0.41 \\
\hline \hline
\end{tabular}

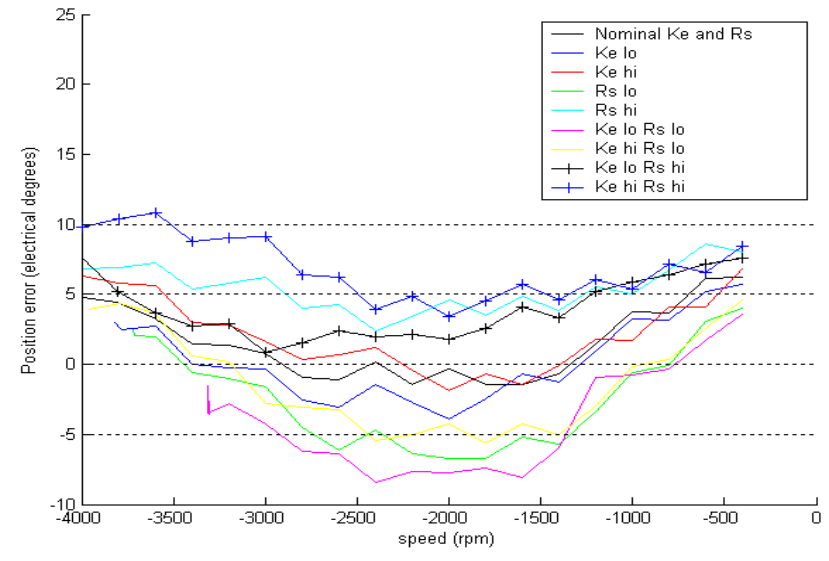

Fig. 11. Position errors at various operating speeds, subject to parameter variation of $R_{s}$ and $K_{e}$ about their nominal value.

\section{Robustness study}

Observer robustness when subject to parameter variations is an essential feature of candidate estimation schemes when motors are manufactured in high volumes, where parameters such as the phase resistance, $R_{s}$, and the back-emf constant, $K_{e}$, in particular, can change significantly due to variations in ambient temperature. A number of trials have therefore been undertaken to investigate the impact of parameter variations on estimates of rotor position, over a wide speed range (including field weakening regime, $i_{d} \neq 0$ ). The results, which have been taken for both the nominal parameters, and $\approx 20 \%$ variations in $R_{s}$ and $\approx 11 \%$ variations in $K_{e}$, (Table II) are given in Fig. 11. For each selected case, it can be seen that the observer performance remains stable and the maximum rotor position error is $\approx 12^{\circ}$-well within the accuracy requirements for this application. Notably, the worst-case error is evident upon occurrences of both extreme variations of $K_{e}$ and $R_{s}$.

\section{CONCLUSION}

Two observer-based rotor position estimation schemes for sensorless control of IPMSMs for an automotive electric power steering system, have been investigated. The first employs a linearised controller with the second scheme considering a complete non-linear implementation. For the latter, difficulties in tuning the observer gain terms have prompted an investigation into the use of parametric 'sweep' tests and GAs, with tuning based on GAs showing a marked performance improvement.

Importantly, for cost critical applications, it is highly desirable that such observer schemes make use of existing processing hardware. In this case, computational overhead and memory requirements are of significant importance. Table III therefore shows the resource requirement for both techniques, with a corresponding EKF implementation (a traditional solution to such problems) being included as a benchmark. In this case, the feedback linearisation/Luenberger observer technique is seen to provide the highest resource efficiency. 


\section{ACKNOWLEDGMENT}

The authors would like to thank TRW Automotive UK for providing funding for the research, with particular thanks to Drs. Connel Williams and Jeff Coles for their valuable contributions.

\section{REFERENCES}

[1] Electric Steering Systems - E-Steer ${ }^{\mathrm{TM}}$ electric power steering. Delphi.

[2] J.X Shen, Z.Q. Zhu and D. Howe "Improved speed estimation of sensorless PM brushless AC drives" IEEE Trans. Ind. Electronics, vol. 38(4), pp.1072-1080.

[3] A. Bado, S. Bologani, M. Zigliotto "Effective estimation of Speed and Rotor Position of an PM Synchronous Motor Drive by a Kalman Filtering Technique" IEEE Proc. PESC, 1992, pp.951-957.

[4] G. Zhu, A. Kaddouri, L. Dessiant and O. Akhrif "A Nonlinear State Observer for the Sensorless Control of a Permanent-Magnet AC Machine" IEEE Trans. Ind. Electronics, vol. 48, 2001, pp.1098-1108.

[5] D. Hamada, K. Uchida, L. F, Yusivar, S. Wakeo and T. Onuki "Sensorless Control of PMSM using a Linear Reduced Order Observer including Disturbance Torque Estimation” EPE'99 - Lausanne, Waseda University.

[6] J. Solsona and C. Muravchik "A Nonlinear Reduced Order Observer for Permanent Magnet Synchronous Motors" IEEE Trans. Ind. Electronics, vol. 43, 1996, pp.492-497.

[7] R. Dhaouadi, N. Mohan and L. Norum, "Design and Implementation of an Extended Kalman Filter for the State Estimation of a Permanent Magnet Synchronous Motor" IEEE Trans. on Power Electronics, vol. 6, 1999, pp.491-497.

[8] S. Bolognani, R. Oboe and M. Zigliotto, "Sensorless Full-Digital PMSM Drive With EKF Estimation of Speed and Rotor Position" IEEE Trans. Ind. Electronics, vol. 46, 1999, pp.184-191.

[9] B. Bhangu, C. Williams, C. M. Bingham and J. Coles "EKFs and other Nonlinear State-Estimation Techniques for Sensorless Control of Automotive PMSMs"; Proc. SPEEDAM 2002, pp.C5 33-38, Italy.

[10] B. Bhangu, C. M. Bingham, "Nonlinear State-Observer Techniques for Sensorless Control of Automotive PMSM's, including Load-Torque Estimation and Saliency"; EPE 2003, Toulouse, France.

[11] N. Matsui and M. Shigyo, "Brushless dc Motor Control without Position and Speed sensors" IEEE Trans. Ind. Electron., vol. 28, 1992, pp. 120127.

[12] B. Bhangu "Sensorless control of PMSMs for automotive electric power steering systems" Thesis, Sheffield University, 2004.

[13] L. Feng, Y. Qiwen, "Applications of GAs in Sensorless Speed adjustment Control for Induction Motor Drive System" Power Electronics and Motion Control Conf., Proc. PIEMC 2000, vol. 1, pp. 296-301. 